\title{
Innovación y desarrollo de sistema ArduinLSPC para implementación en sistemas hidráulicos de maquinaria pesada
}

\section{Innovation and development of ArduinLSPC system for implementation in hydraulic systems of heavy machinery}

BANDERAS-MENDOZA, Alexis Alfredo $\dagger^{*}$, RAMIREZ-ALONSO, Daniel Martin, JIMENEZVARGAS, Saúl y GARCÍA-MARTINEZ, Erick Oswaldo

Universidad Tecnológica de Jalisco, Guadalajara, Jalisco, México

ID $1^{\text {er }}$ Autor: Alexis Alfredo, Banderas-Mendoza / ORC ID: 0000-0002-8723-025X, CVU CONACYT ID: 1015929

ID $1^{\mathrm{er}}$ Coautor: Daniel Martin, Ramirez-Alonso / ORC ID: 0000-0002-5163-5087, CVU CONACYT ID: 1021378

ID $2^{\text {do }}$ Coautor: Saúl, Jimenez-Vargas / ORC ID: 0000-0003-2191-8972, CVU CONACYT ID: 1021373

ID $3^{\text {er }}$ Coautor: Erick Oswaldo, García-Martinez / ORC ID: 0000-0001-7388-655X, CVU CONACYT ID: 1021370

DOI: $10.35429 /$ JIE.2019.7.3.25.28

Recibido 25 de Enero 2019; Aceptado 28 Marzo, 2019

\begin{abstract}
Resumen
Este articulo presenta una innovación en el tema Industria 4.0 enfocado en los sistemas hidráulicos LSPC, a partir del análisis de una clase práctica con dicho sistema; este nuevo sistema implementa un diseño más compacto y eficiente, logrando mayor eficacia y una entrega de fluido proporcional requerida por el sistema. Para lograr este proyecto se está aprovechando los conocimientos de las materias de electrónica digital y sistemas Neumáticos e hidráulicos; que los alumnos llevan a lo largo de su carrera de TSU. En Mantenimiento Area Maquinaria Pesada.
\end{abstract}

Industria 4.0, ArduinLSPC, Maquinaria Pesada

\begin{abstract}
This article presents an innovation in the topic Industry 4.0 focused on hydraulic systems LSPC, from the analysis of a practical class with this system; this new system implements a more compact and efficient design, achieving greater efficiency and a proportional fluid delivery required by the system. To achieve this project is taking advantage of the knowledge of the subjects of digital electronics and Pneumatic and hydraulic systems; that the students carry throughout their TSU career. In Heavy Machinery Area Maintenance.
\end{abstract}

Industry 4.0, ArduinLSPC, Heavy Machinery

Citación: BANDERAS-MENDOZA, Alexis Alfredo, RAMIREZ-ALONSO, Daniel Martin, JIMENEZ-VARGAS, Saúl y GARCÍA-MARTINEZ, Erick Oswaldo. Innovación y desarrollo de sistema ArduinLSPC para implementación en sistemas hidráulicos de maquinaria pesada. Revista de Ingeniería Industrial. 2019. 3-7: 25-28

\footnotetext{
* Correspondencia del Autor (AlfredoBanderas10@ hotmail.com)

$\dagger$ Investigador contribuyendo como primer autor.
} 


\section{Introducción}

La primera vez que vimos el funcionamiento de los sistemas hidráulicos LSPC, observamos que este mismo tenía diferentes deficiencias en la entrega de fluido a los cilindros. El profesor nos mencionó sobre la utilidad del arduino y nos cuestionó ¿Cómo podemos aplicar estas tecnologías en nuestra área de maquinaria pesada? $\mathrm{O}$ ¿Cómo innovar algún sistema de una maquinaria pesada? A partir de estas dos interrogantes surgió el proyecto ArduinLSPC, el cual pretende innovar en la industria 4.0 en el área de hidráulica, ya que dicho sistema busca ser más eficiente en la entrega de fluido a través de electroválvulas accionadas por medio de señales eléctricas digitales que controlarán la entrada de fluido proporcional a los cilindros.

\section{Definición del problema}

En la actualidad, los sistemas LSPC son ineficientes para el uso general de un sistema hidráulico con más de un cilindro.

El principal problema que se tiene en dicho sistema es que, al poner dos cilindros de cargas externas diferentes en un mismo circuito, el sistema LSPC manda la presión hidráulica al cilindro con menor carga dándole prioridad y consecuentemente al de mayor carga.

\section{Hipótesis}

Con tecnología e información de fácil acceso y los conocimientos que se le dan a los alumnos de la carrera de maquinaria pesada es posible lograr implantar en un sistema LSPC señales analógicas convirtiéndolas a eléctricas digitales.

\section{Justificación}

Desarrollar nuevas competencias e innovar la industria 4.0 en área de hidráulica, ofreciendo de esta manera un desarrollo en la comunicación de señales analógicas a eléctricas digitales.

\section{Objetivo}

Diseñar y desarrollar un sistema LSPC que logré combinar señales analógicas y eléctricas digitales; dar una dosificación precisa, reducir mantenimientos y costos de operación, detectar anomalías y generar un diagnostico eficaz.
Aportar un avance en la industria 4.0 al combinar un sistema analógico con un sistema digital.

Combinar las nuevas tecnologías en sistemas de maquinaria pesada.

Para lograr este proyecto se está aprovechando los conocimientos de las materias de electrónica digital y sistemas Neúmaticos e Hidráulicos; dichas clases impartidas en la carrera de Mantenimiento Área Maquinaria Pesada.

\section{Importancia}

Hoy en día la maquinaria pesada es la rama industrial que mas recursos utiliza, una de la que más genera, pero también la que más contamina el medio ambiente, por lo cual, nuestro proyecto ofrece una de las bases para crear maquinaria pesada más eficiente. Podrían crearse maquinas con las mismas capacidades e incluso mayores, pero con un tamaño más compacto, por ende, a menor masa que mover, menor cantidad de energía necesaria para realizar el trabajo, por ende, se traduce a menor tiempo de trabajo, menores costos de mantenimiento y menor consumo de combustible.

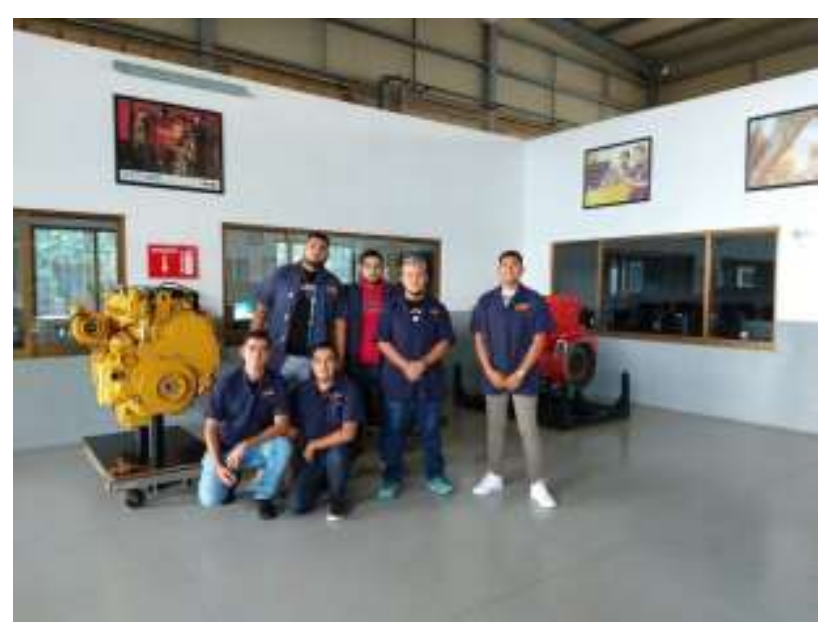

Figura 1 Alumnos Mantenimiento Área Maquinaria Pesada (12 de Julio, 2019)

Fuente: Propia, capturada en el taller de Maquinaria Pesada de UTJ

\section{Método}

Para poder diseñar y desarrollar el proyecto los alumnos participantes se están apoyando en el conocimiento adquirido en las materias de electrónica digital, sistemas neumáticos $\mathrm{y}$ hidráulicos. 
En lo referente a la etapa de control electrónico conformada por el sistema arduino que es una plataforma de hardware libre, basada en una placa con un micro -controlador y un entorno de desarrollo, diseñado para facilitar el uso de la electrónica en proyectos (John, 2013), multidisciplinares que por su facilidad es una buena opción para el desarrollo de la etapa de controlar el flujo de caudal, que se determinó como objetivo del ArduinLSPC, usando la implementación de un sensor de presión HBM.

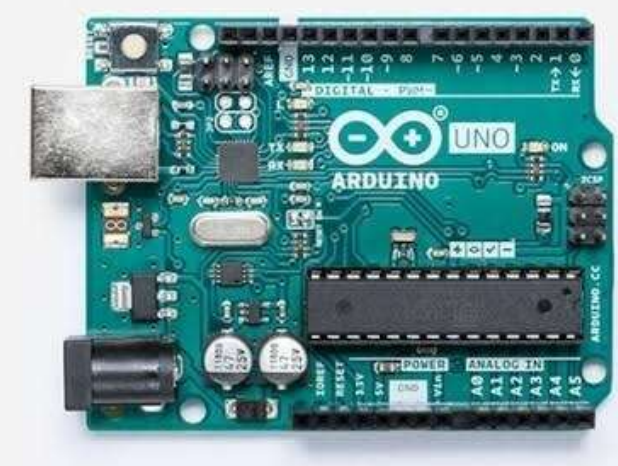

Figura 2 Arduino

Fuente:https://www.xataka.com/ecommerce/que-comprar -leer-para-empezar-arduino

En la etapa de transmisión de señales el sensor HBM será el encargado de la presión señal (analógica) la cual enviará la lectura al arduino y este mismo enviará señales eléctricas digitales de voltaje controlado a los selenoides suministrando a la vez el voltaje para activar las electroválvulas, mismas que controlarán el flujo de caudal, de esta manera al ser activadas por dichas señales las electroválvulas que permitirán el paso del fluido tomarán su posición correspondiente de la presión señal y en efecto los pistones recibirán un caudal proporcional, evitando vibraciones en la tubería, remplazando el acumulador; y para mayor eficiencia, la bomba de pistones axiales cuenta de igual manera con una electroválvula para el control del plato basculante.

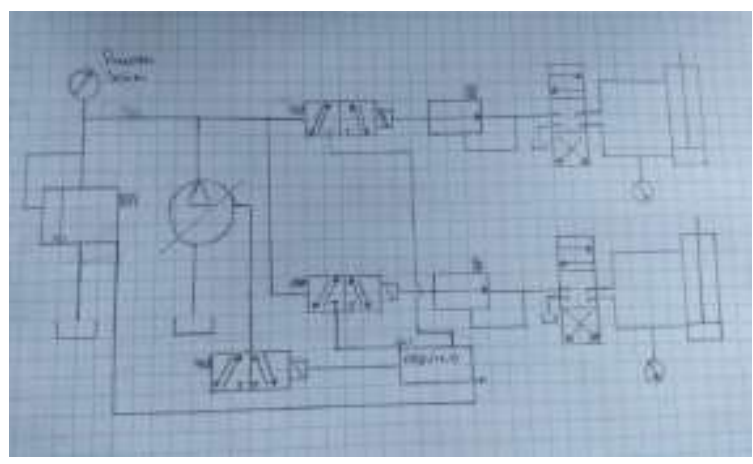

De está manera se logra reducir el tamaño del sistema porque se sustituye la línea de drenaje de fluido en la sección de las válvulas compensadoras, ya que en dicha sección se localiza una válvula limitadora de presión y al ser accionada activa el retorno del fluido al tanque.

Si no tenemos está sección se concentrará la presión en las lineás principales, que van desde la bomba de caudal variable hasta los cilindros.

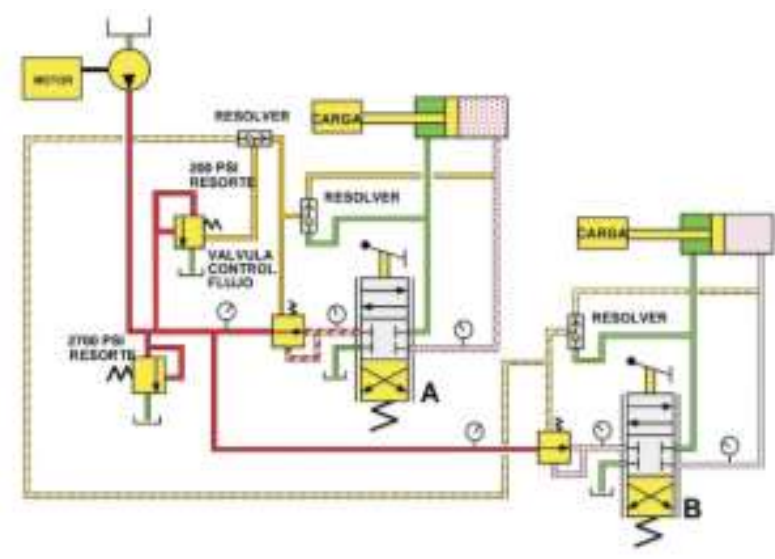

Figura 4

Fuente: Manual de hidráulica básica Caterpillar

\section{Resultados}

Los resultados esperados son que al combinar un sistema hidráulico con el arduino (Señales eléctricas digitales) lograremos un sistema compacto, con mayor precisión, eficaz en el control de presión y flujo de caudal.

Además, mejorando áreas de oportunidad como las vibraciones por golpes de presión, reducción de mantenimientos y control simultaneo de los cilindros.

\section{Conclusiones}

Somos estudiantes de cuarto cuatrimestre, por ende, planeamos desarrollar aún más nuestro sistema. Todavía falta bastante por construir del ArduinLSPC, sin embargo, se espera que en los próximos cuatrimestres restantes se complete el proyecto $y$ en el futuro implementar el desarrollo la transmisión de datos a una base donde se pueda manipular manualmente por el operador efectuando de esta manera un mayor control del sistema para ser más eficaz en las operaciones solicitadas.

Figura 3 Diagrama de ArduinLSPC

Fuente: Elaboración Propia 


\section{Referencias}

Pallás Areny, R. (2007). Sensores acondicionadores señal. 4th ed. México D.F: Alfaomega grupo editor.

Miguel Reyes Aguirre. (1998). Máquinas Hidráulicas. México, D.F: Alfaomega

H. W. King y E. F. Brater. (1962). Manual de hidráulica. México 12, D.F.: Unión Tipográfica editorial hispano-americana.

Pablo R. Cardoso Ponce de León. (1993). Manual de prácticas de hidráulica. México, D.F: Universidad autónoma metropolitana unidad Iztapalapa.

Hubert Chanson . (2002). Hidráulica del flujo. Bogotá, D.C., Colombia : Mc Graw Hill.

Sybil P. Parker. (1990). Diccionario McGraw Hill de ingeniería Mecánica y Diseño. Naucalpan de Juárez, Eso. de México: Mc Graw Hill. 